\title{
Limited adaptation to non-natal osmotic environments at high water temperature in euryhaline wanderer fishes
}

\author{
Masahiro Nakamura (D) - Soichi Watanabe • \\ Toyoji Kaneko $\cdot$ Reiji Masuda $\cdot$ Katsumi Tsukamoto • \\ Tsuguo Otake
}

Received: 2 April 2019 /Accepted: 25 November 2019/Published online: 6 December 2019

(C) The Author(s) 2019

\begin{abstract}
Gradual transfer experiments were conducted to see whether Japanese dace Tribolodon hakonensis (freshwater species) and Japanese black porgy Acanthopagrus schlegelii (marine species) could acclimate to high and low temperatures in natal and nonnatal osmotic environments. Two groups of each species, one acclimated to their natal osmotic environment and the other to a non-natal osmotic environment, were exposed to gradual temperature changes. Under high temperature conditions, the plasma osmolality (Posm) of both species approached the osmolality of ambient water, and high mortality was observed in the non-natal
\end{abstract}

Electronic supplementary material The online version of this article (https://doi.org/10.1007/s10641-019-00940-0) contains supplementary material, which is available to authorized users.

M. Nakamura $(\bowtie) \cdot$ S. Watanabe $\cdot$ T. Kaneko •

K. Tsukamoto $\cdot$ T. Otake

Graduate School of Agricultural and Life Sciences, The University of Tokyo, Bunkyo, Tokyo 113-8657, Japan

e-mail: mnakamura@affrc.go.jp

M. Nakamura

National Research Institute of Fisheries and Environment of Inland Sea, Fisheries Research Agency, Imabari, Ehime 794-2305, Japan

\section{R. Masuda}

Maizuru Fisheries Research Station, Field Science Education and Research Center, Kyoto University, Nagahama, Maizuru, Kyoto 625-0086, Japan

K. Tsukamoto

College of Bioresource Sciences, Nihon University, Kameino,

Kanagawa 252-0880, Japan osmotic environments but not in the natal osmotic environments. In contrast, there was no clear evidence that the osmoregulatory capacity of either species had declined under low temperature conditions in the natal and non-natal osmotic environments. Limited adaptation of these fishes to non-natal osmotic environments at high temperature suggests that global warming may impair their entry to non-natal habitats.

Keywords Euryhaline wanderer - Global warming . Non-natal osmotic environment . Plasma osmolality . Temperature

\section{Introduction}

Euryhaline wanderer fishes in the temperate zone are known to exhibit seasonal habitat changes (Day et al. 1989; Able and Fahay 1998; Ishitobi et al. 2000; Able and Fahay 2010). These species typically enter nonnatal osmotic environments (hypertonic environments for freshwater species and hypotonic environments for marine species) in estuaries during spring to summer and migrate back to their natal osmotic environments during fall and winter (Nakamura et al. 2016). Since these species typically enter estuaries in warm seasons, coastal warming, which is occurring across broad areas of the world's coastlines (Lima and Wethey 2012), may force them to tolerate higher temperatures in non-natal osmotic environments. This may be problematic because the osmoregulatory capacities of euryhaline fishes can be affected by water temperature (Sardella et al. 
2008; Nakamura et al. 2016; Masroor et al. 2018; Vargas-Chacoff et al. 2018). However, only a few studies have investigated the relationship between osmoregulatory capacity and temperature in terms of euryhaline wanderers' natal and non-natal osmotic environments (Nakamura et al. 2016; Masroor et al. 2018), and the relationship between these factors remains unclear.

A recent study on two euryhaline wanderers, Japanese dace Tribolodon hakonensis (freshwater species) and Japanese black porgy Acanthopagrus schlegelii (marine species), reported that they are less tolerant of abrupt temperature changes in non-natal osmotic environments than in their natal osmotic environments (Nakamura et al. 2016). Dace inhabit areas from the upper reaches of rivers to coastal bays (Moriyama et al. 2008; Ishizaki et al. 2009) whereas black porgy is found in habitats from coastal areas to the upper reaches of estuaries (Fujita 2004; Tran et al. 2019). Both species are widely distributed in the temperate regions of Japan, which have wide temperature ranges throughout the year, so they can experience a wide range of water temperatures including summer temperatures of around $30{ }^{\circ} \mathrm{C}$ and winter temperatures of around $10{ }^{\circ} \mathrm{C}$ (Fujita 2004; Tsutsumi and Guo 2016). Nakamura et al. (2016) exposed these species to temperature changes of up to $13{ }^{\circ} \mathrm{C}$ within $3 \mathrm{~h}$, and determined their capacity to acclimate to high and low temperatures in a short period of time. This study suggested that temperature increases in estuaries can possibly influence the seasonal habitat transition of dace and black porgy that enter estuaries during summer (Sakai 1995; Katano et al. 2010; Yokoo et al. 2010). In general, however, fish are able to become acclimated to a broader range of temperatures when subjected to gradual temperature changes than when subjected to sudden temperature changes (Bennett and Judd 1992; Bennett and Beitinger 1997). Thus, in order to estimate the potential effect of global warming on the migratory ecology of euryhaline wanderers, the capacity to acclimate to high and low temperatures needs to be validated under gradual temperature changes.

The objective of this study was thus to empirically compare the range of temperatures to which dace and black porgy could acclimate when they were subjected to gradual temperature changes in their natal and nonnatal osmotic environments. Firstly, two groups of each species, one acclimated to their natal osmotic environment and the other to a non-natal osmotic environment, were prepared at a water temperature of $20^{\circ} \mathrm{C}$. Next, the fish in each group were divided into hot, cool and control groups in the osmotic environment to which they had been acclimated. The fish in the hot and cool treatments were exposed to temperature increases and decreases, respectively, of $1{ }^{\circ} \mathrm{C}$ per day (challenge tests). The control group was maintained at $20{ }^{\circ} \mathrm{C}$ throughout the 15-day experimental period. Fish were sampled at regular intervals during this period, and the plasma osmolality (Posm) of individuals and mortality were determined. The Posm data obtained from the hot and cool treatments were applied to a generalized linear model (GLM) or quadratic curve and compared with the corresponding data obtained from the control groups, in order to estimate whether the osmoregulatory capacity was maintained.

\section{Materials and methods}

\section{Animals}

The Japanese dace used in this study were collected in 13 January 2014 with a throw net in the pure freshwater area of the Terakawa River $\left(35^{\circ} 28^{\prime} 15 \mathrm{~N}, 135^{\circ} 23^{\prime} 33 \mathrm{E}\right)$. After being captured, the individuals were transported to the Maizuru Fisheries Research Station (MFRS) of Kyoto University located in Maizuru (35'29’21 N, $\left.135^{\circ} 22^{\prime} 03 \mathrm{E}\right)$. The individuals used in the experiments were fingerings and ranged in standard length (SL) from 80 to $162 \mathrm{~mm}$. The Japanese black porgy used in this study were hatchery-born and reared in filtered seawater at the MFRS under a natural photoperiod and water temperature. The individuals used in the experiments were fingerings and ranged in SL from 59 to $104 \mathrm{~mm}$. Since these species start entering estuaries in the late larval or juvenile stage (Fujita 2004; Ishizaki et al. 2009; Tran et al. 2019), the individuals of both species used in the experiments were within the migratory size range.

About 250 dace and 150 black porgy were kept in 500-1 clear polycarbonate tanks (two tanks for dace and one for black porgy) filled with freshwater and seawater, respectively. Water temperature was not adjusted in all the tanks. The fish were fed to satiation with commercial pellets (dace: Kawasakana-no-esa, GEX, Japan; black porgy: Otohime S2, Marubeni Nisshin Feed, Japan) once a day for about six days until being transported to the University of Tokyo (35 $\left.42^{\prime} 59 \mathrm{~N} ; 1^{\circ} 9^{\circ} 45^{\prime} 37 \mathrm{E}\right)$ on 20 January 2014. After being transported to the University of Tokyo, the dace and porgy were introduced into 700-1 FRP tanks filled with well-aerated 
$20{ }^{\circ} \mathrm{C}$ freshwater and seawater, respectively. The SW used at the University of Tokyo was surface water of the Kuroshio Current flowing offshore in the northwestern Pacific. Those two tanks were equipped with filtering and temperature control systems. The tanks were located outdoors, and were covered with an open roof that exposed the fish to a natural photoperiod. The fish were fed to satiation once a day with the commercial pellets mentioned above.

\section{Acclimation of fish}

To prepare the natal osmotic environment-acclimated groups, about half of the total fish (120 dace and 70 black porgy) were kept in the original 700-1 FRP tanks for 2-3 months. To prepare the non-natal osmotic environment-acclimated groups, the rest of the dace and porgy were acclimated in FRP tanks through several steps at $20{ }^{\circ} \mathrm{C}$ as follows, since direct transfer to nonnatal osmotic environments can cause high mortality. For acclimating the dace to seawater, they were first transferred from freshwater to $15 \mathrm{ppt}$ seawater. Five days after this first transfer, they were transferred to full-strength seawater (32 ppt). The black porgy were first transferred from full-strength seawater to $10 \mathrm{ppt}$ seawater. After an acclimation period of one day, the salinity of the tank water was reduced to $5 \mathrm{ppt}$, followed by a 2 ppt degradation each day until the salinity reached $1 \mathrm{ppt}$. The fishes were kept there for more than 10 days and treated as non-natal osmotic environmentacclimated groups (dace: seawater-acclimated (SWacclimated) group; black porgy: hypotonic environment-acclimated (HE-acclimated) group). The black porgy were acclimated to a hypotonic environment of 1 ppt instead of freshwater since high mortality was observed in freshwater in an exploratory experiment.

\section{Procedure of challenge tests}

Three 300-1 acrylic tanks equipped with filtering and temperature control systems were used for each challenge test conducted from February 14 to May 13 of 2014. The three tanks were used as hot, cool and control groups. The experiments were conducted consecutively for the SW-acclimated dace, SW-acclimated black porgy, freshwater (FW)-acclimated dace and HEacclimated black porgy. The water temperatures of the hot and cool treatments were raised and lowered, respectively, $1{ }^{\circ} \mathrm{C} /$ day from $20^{\circ} \mathrm{C}$ with the fish in the tanks. The control tank was maintained at $20^{\circ} \mathrm{C}$. Since the experimental period was 15 days, the temperatures of the hot and cool treatments were expected to reach $35^{\circ} \mathrm{C}$ and $5{ }^{\circ} \mathrm{C}$, respectively, by the end of the experimental period. However, the highest temperatures of the hot treatments of the SW- and FW-acclimated dace were $31^{\circ} \mathrm{C}$ and $32{ }^{\circ} \mathrm{C}$, respectively, due to high mortality. The lowest temperatures of the cool treatments of the HEacclimated porgy and the $\mathrm{FW}$-acclimated dace were $6{ }^{\circ} \mathrm{C}$ and $7{ }^{\circ} \mathrm{C}$, respectively, since the capability of the temperature cooling system was limited. The water temperatures of the other treatments were successfully changed in accordance with the experimental design (Table S1, S2).

At the beginning of each challenge test, five individuals were sampled from each acclimation tank as initial samples. After this, the following number of individuals were put into each of the three 300-1 tanks mentioned above: 30 individuals of the SW-acclimated dace, 30 individuals of the FW-acclimated dace, 20 individuals of the SW-acclimated porgy, and 18 individuals of the HE-acclimated porgy. The number of individuals of the HE-acclimated black porgy was smaller than that of the SW-acclimated porgy because the mortality of the black porgy during the HE-acclimation period was so high that we could not prepare enough individuals. Five individuals of the dace and black porgy were sampled every three and five days, respectively, as long as enough individuals were left in the tanks. Dead individuals were removed from the tanks every day. Due to high mortality, only four individuals could be sampled at the last sampling point for the control and hot treatment of the HE-acclimated black porgy and at the fourth sampling point for the hot treatment of the SWacclimated dace (Table S1, S2). Fish were not fed during the challenge tests.

\section{Sampling}

Sampled individuals were anesthetized with $0.1 \%$ 2phenoxyethanol, and standard length $\left(L_{\mathrm{S}}\right)$ and wet mass $\left(M_{\mathrm{W}}\right)$ were measured. Blood was collected from caudal vessels with a heparinized syringe and needle. Blood plasma was separated by centrifugation $(5000 \mathrm{rpm}$, $5 \mathrm{~min}$ ) and stored at $-20{ }^{\circ} \mathrm{C}$. Later, the Posm was measured with a vapor pressure osmometer (Wescor 5520, Logan, UT, USA). 
Statistical analysis

All the statistical procedures were conducted using the statistical software R version 3.4.1 (R Core Team 2017). The Posm of each of the hot and cool treatments was plotted against the water temperature and compared with the Posm of the control sampled at approximately the same time to determine if the osmoregulatory capacity of each species was maintained. We used generalized linear models (GLM) for the hot and cool treatments to define the relationship between the Posm and the water temperature, assuming a gamma error distribution and a $\log$ link function. For the cool treatment of the HEacclimated black porgy, the Posm showed a peculiar curve, and the GLM showed a poor fit (AIC: 173.8). Thus, for this treatment, data were fit to a quadratic curve using nonlinear least squares estimates (AIC: 166.6). Whether osmoregulatory ability had decreased by the effect of temperature change was estimated by comparing the Posm at a given temperature with the Posm of the control at the nearest time point. Survival rates were calculated by the following equation:

survival rate $(\%)=\left(\frac{i-d}{i}\right) \times 100$

where $i$ is the number of individuals introduced in each tank and $d$ is the cumulative number of dead individuals. The significance of these differences was determined by Welch's $t$-tests. The "ggplot2 version 3.0.0" package was used for model fitting and generating figures. The significance level of all the statistical tests was set at $\alpha=0.05$.

\section{Results}

Experiment 1: temperature challenge on dace

In FW, the Posm decreased slightly as the ambient water temperature decreased or increased and was significantly lower than the Posm of the control at $32{ }^{\circ} \mathrm{C}$ and at every temperature lower than $14{ }^{\circ} \mathrm{C}$ (Welch's $t$-test, $P<0.05)$ (Fig. 1a). However, the estimated plasma osmolalities at the highest $\left(32{ }^{\circ} \mathrm{C}\right)$ and lowest $\left(7{ }^{\circ} \mathrm{C}\right)$ temperatures were only 2-3\% lower than the estimated Posm at $20^{\circ} \mathrm{C}$, and the range of variation in the Posm was much smaller than that in SW (Fig. 1, Table S1).
In SW, which is a non-natal osmotic environment for this species, the Posm showed a significant increase in association with the increase in ambient water temperature, and the estimated plasma osmolality at $31^{\circ} \mathrm{C}$ was $13 \%$ higher than that at $20^{\circ} \mathrm{C}$ (Fig. 1b). The Posm at every temperature above $26{ }^{\circ} \mathrm{C}$ was higher than the Posm of the control (Welch's $t$-test, $P<0.01$ ). In the cool treatment, the Posm decreased gradually as the ambient water temperature decreased, and the estimated plasma osmolality at $5{ }^{\circ} \mathrm{C}$ was $4 \%$ lower than that at $20{ }^{\circ} \mathrm{C}$. The Posm at $8{ }^{\circ} \mathrm{C}$ was significantly lower than the Posm of the control (Welch's $t$-test, $P=0.02$ ).

In FW, no individuals died in the cool treatment and control, whereas ten individuals died in the hot treatment when the temperature reached $32{ }^{\circ} \mathrm{C}$. High mortality was observed in the $\mathrm{SW}$-acclimated group. In total, six individuals in the cool treatment and control, and eleven individuals in the hot treatment died (Fig. 2, Table S1).

Experiment 2: temperature challenge on porgy

In HE, which is a non-natal osmotic environment for this species, no difference was observed between the Posm of the control and the Posm of the hot treatment until the temperature in the hot treatment reached $30^{\circ} \mathrm{C}$. However, when the temperature reached $35^{\circ} \mathrm{C}$, the Posm showed a marked drop and became significantly lower than the Posm of the control (Welch's $t$-test, $P=$ 0.02) (Fig. 3a). The estimated plasma osmolality at $35^{\circ} \mathrm{C}$ was $12 \%$ lower than that at $20{ }^{\circ} \mathrm{C}$. The Posm of the cool treatment consistently decreased as the ambient water temperature got lower, and the estimated plasma osmolality at $6{ }^{\circ} \mathrm{C}$ was $9 \%$ lower than that at $20^{\circ} \mathrm{C}$. The Posm at $10{ }^{\circ} \mathrm{C}$ and $6{ }^{\circ} \mathrm{C}$ were significantly lower than the Posm of the control (Welch's $t$-test, $P<0.05$ ).

In SW, which is the natal osmotic environment for this species, the Posm of the hot treatment did not differ from that of the control at any sampling point (Welch's $t$ test, $P>0.33$ ) (Fig. 3b). In contrast, the Posm of the cool treatment decreased in the same manner as that of the HE cool treatment, and the estimated plasma osmolality at $5{ }^{\circ} \mathrm{C}$ was $9 \%$ lower than that at $20{ }^{\circ} \mathrm{C}$. The Posm at $5{ }^{\circ} \mathrm{C}$ was significantly lower than the Posm of the control (Welch's $t$-test, $P=0.003$ ).

In HE, four individuals died in each of the hot treatment and control group, whereas none died in the cool treatment. In SW, one individual died in the control, 
Fig. 1 Plasma osmolality (Posm) of Japanese dace Tribolodon hakonensis acclimated to natal (freshwater, a) and non-natal (seawater, b) osmotic environments under various temperature conditions. Blue and red indicate cool and hot treatments, respectively. Each colored plot shows the mean Posm at each sampling point. The dashed and solid lines are model fits to data of the cool and hot treatments obtained by the generalized linear model (GLM). Square plots show Posm of the control group, which was determined at approximately the same time as those of the hot and cool treatments. Vertical lines on symbols indicate standard errors (SE). Asterisks indicate significant differences between the hot or cool treatment and the control group at each sampling point (Welch's $t$ test, $* * P<0.01, * P<0.05$ ) (a)

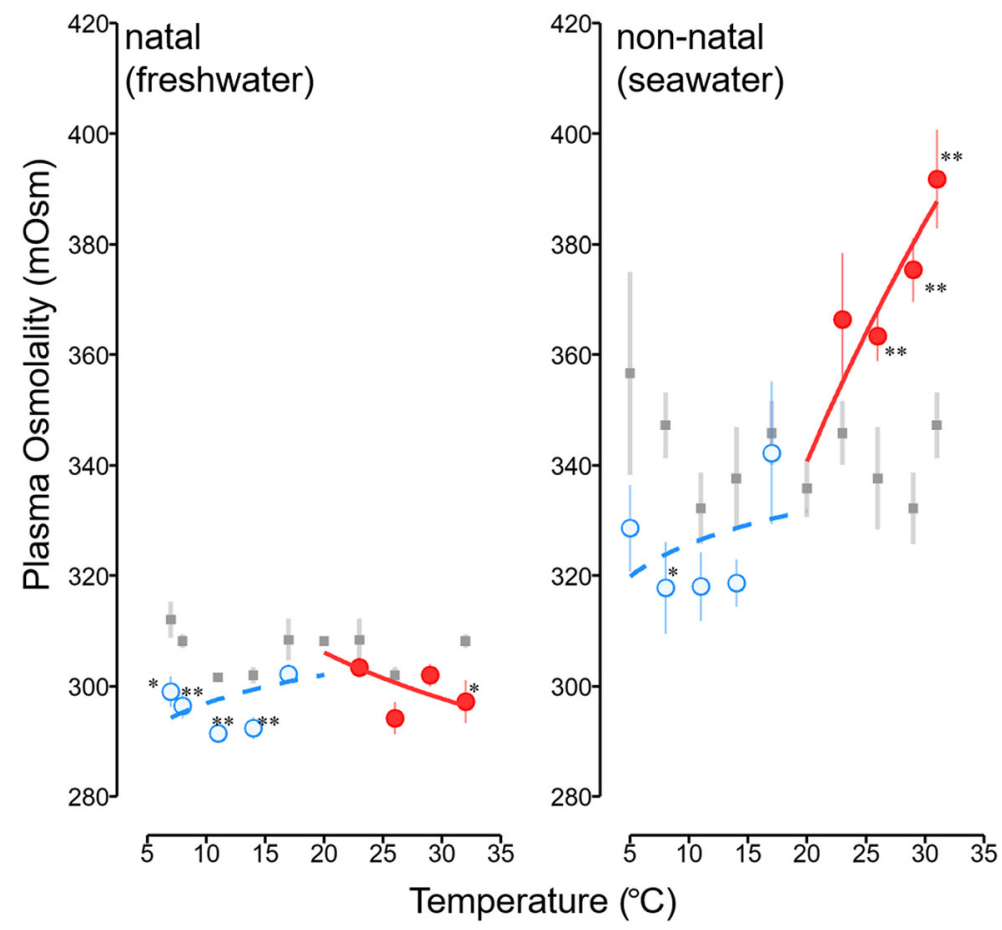

which was the only mortality observed in this group (Fig. 4, Table S2).

\section{Discussion}

Osmoregulatory capacity at high temperature

Whether osmoregulatory capacity was maintained can be estimated by observing the dynamics of the Posm in relation to ambient water osmolality. The Posm of the SW-acclimated dace increased significantly with the increase in temperature. Moreover, the Posm of the HE-acclimated black porgy showed a significant drop at $35^{\circ} \mathrm{C}$. These results clearly show that the Posm of both species approached the osmolality of ambient water under high temperature conditions in the non-natal osmotic environments. This fact suggests that the osmoregulatory capacities of both species were reduced in the non-natal osmotic environments under warmer conditions. Contrastingly, in the natal osmotic environment for each species, no clear change in the Posm was observed in response to the temperature rise. Although the Posm of the dace at $32{ }^{\circ} \mathrm{C}$ was significantly lower than the Posm of the control, the high Posm in the control, not the decline in the Posm of the hot treatment, seems to be the cause of this result, since the decreasing trend obtained from the model was not so clear and the variation in the Posm between the individuals was small (Fig. 1b, Table S1). These results clearly indicate that the capacities of dace and black porgy to acclimate to high temperatures are lower in non-natal osmotic environments than in their natal osmotic environments. This feature is shared by both these species of freshwater and marine fishes, suggesting that this is a general characteristic of euryhaline wanderers. Both species occur in nature at the salinities of the non-natal osmotic environments tested in this study (Ishizaki et al. 2009; Tran et al. 2019) and extremely hot days are becoming more common in broad areas of the world (Lima and Wethey 2012). These indicate that the entry of euryhaline wanderer fishes into non-natal osmotic environments during warm seasons might be influenced by coastal warming.

\section{Osmoregulatory capacity in low temperature}

The Posm of the dace and the Posm of the black porgy decreased as the temperature got lower both in the natal 
Fig. 2 Survival rate of Japanese dace Tribolodon hakonensis acclimated to natal (freshwater, a) and non-natal (seawater, b) osmotic environments under various temperature conditions. Blue, red and gray indicate cool, hot and control groups, respectively. Hot treatments in the natal and non-natal osmotic conditions were ended on the 12th (a) and 11th (b) days of experiment, respectively, since all the individuals were dead on these dates

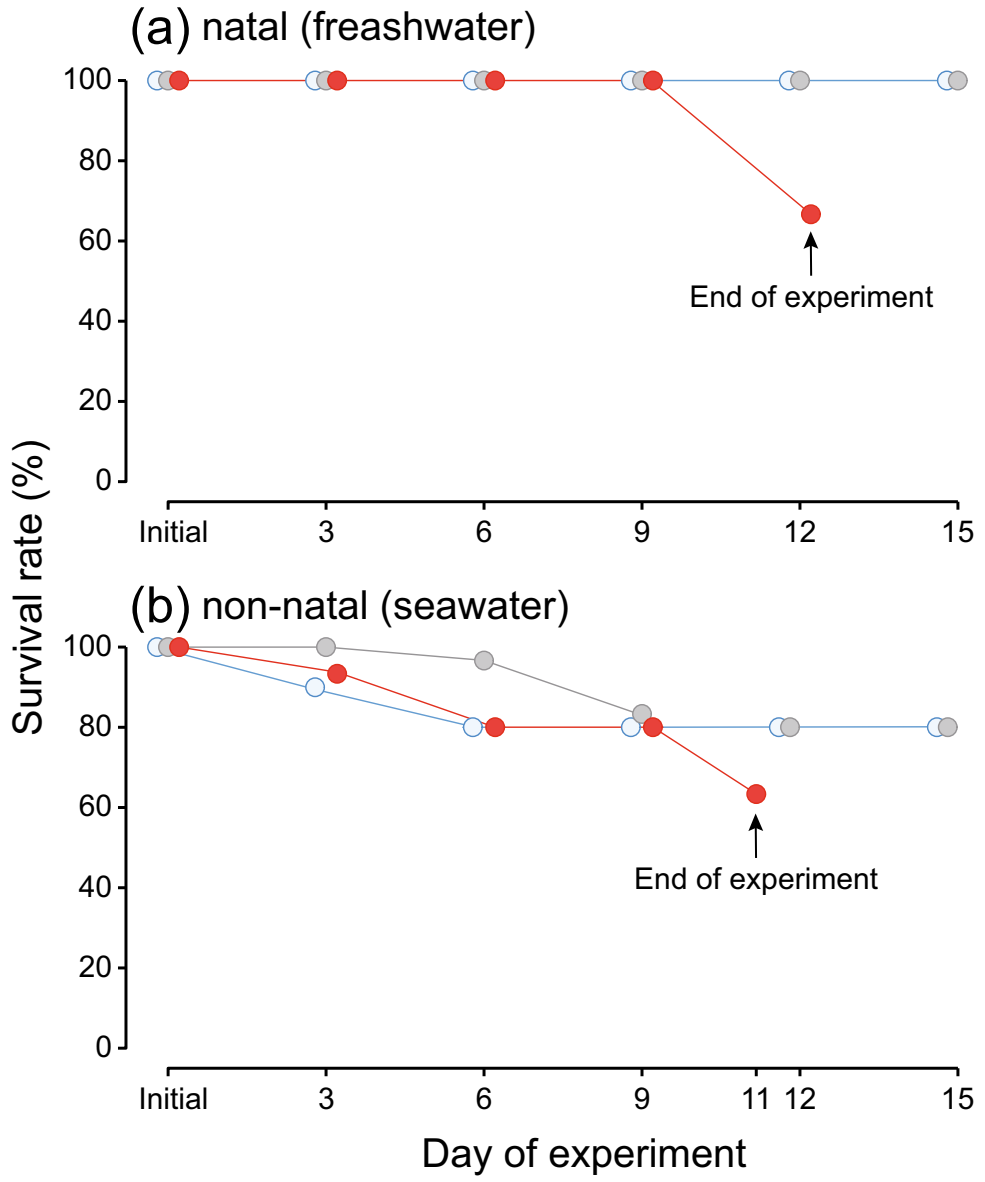

and in non-natal osmotic environments. The cause of the decrease in the Posm of the FW-acclimated dace or HEacclimated black porgy might be an impaired osmoregulatory capacity. However, such an interpretation seems uncertain in the present case for the following reasons. Firstly, the behavior of the Posm of the FWacclimated dace or HE-acclimated black porgy was similar to the behavior of the Posm of the SWacclimated groups, which cannot be associated with a degradation in osmoregulatory capacity. Secondly, the variation in the Posm between the individuals was very small even at the lowest temperature (Tables S1, S2), and no mortality was observed in these treatments (Figs. 2, 4; Tables S1, S2). Thus, it seems more likely that the decrease in the Posm of the FW-acclimated dace or the HE-acclimated black porgy may have been the result of some kind of physiological response to low temperature rather than it being caused by the collapse of the homeostatic mechanism.
Although the FW species and SW species used in our study shared the same characteristics, various patterns in the dynamics of Posm under low temperature conditions have been reported. Several studies as well as our study have reported decreases in the Posm under low temperature in natal or non-natal osmotic environments (Umminger 1971; Finstad et al. 1988). In contrast, some studies have reported increases in the Posm under low temperature (Ahokas and Sorg 1977; Metz et al. 2003; Imsland et al. 2003). Since there is no consistent pattern in dynamics of the Posm under low temperature conditions and the function or mechanism of this Posm decrease/increase remains unclear, further research is needed to clarify why and how the Posm of the dace and the black porgy decreased under the low temperature conditions both in the natal and non-natal osmotic environments.

As described above, our results showed no clear evidence that the osmoregulatory capacity of either species had declined under low temperature conditions in 


\section{(a)}

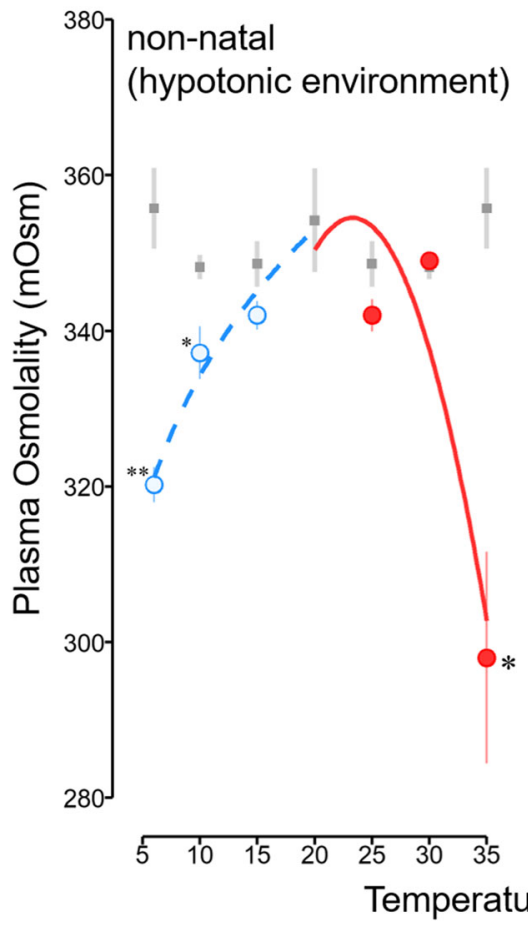

Fig. 3 Plasma osmolality (Posm) of Japanese black porgy Acanthopagrus schlegelli acclimated to non-natal (hypotonic environment, a) and natal (seawater, b) osmotic environments under various temperature conditions. Blue and red indicate cool and hot treatments, respectively. Each colored plot shows the mean Posm at each sampling point. The dashed and solid lines are model fits to data of the cool and hot treatments obtained by the generalized

the natal osmotic environment or the non-natal osmotic environment. This result is different from that obtained in our previous direct transfer experiments, in which many individual dace and black porgy died when exposed to a rapid decrease in temperature in non-natal osmotic environments (Nakamura et al. 2016). The different results obtained from these two studies suggest that both species are capable of acclimating to cold nonnatal osmotic environments when enough time is provided. Nevertheless, experiments under even lower temperatures should be conducted to clarify the lower limit of temperature that they can acclimate to in natal and non-natal osmotic environments. Osmoregulation in cold non-natal osmotic environments may be more costly than that in natal osmotic environments, and this may be a possible cause of migration back to natal osmotic environments in cold seasons. Measuring other parameters such as $\mathrm{Na}^{+} / \mathrm{K}^{+}$-ATPase activity may help evaluate this possibility. (b)

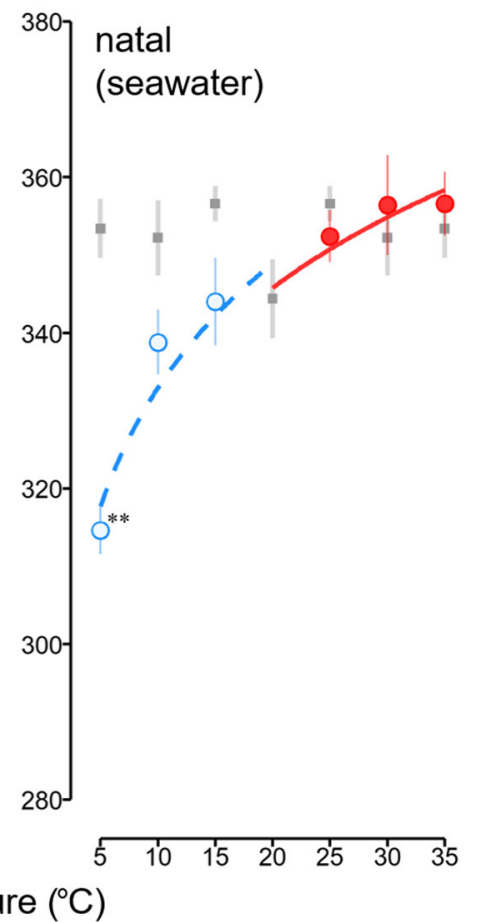

linear model (GLM) or linear least squares regression fits to data points. Square plots show Posm of the control group, which was determined at the same time as those of the hot and cool treatments. Vertical lines on symbols indicate standard errors (SE). Asterisks indicate significant differences between the hot or cool treatment and the control group at each sampling point (Welch's $t$ test, $* * P<0.01, * P<0.05$ )

Our results at high temperature indicate that dace and black porgy may avoid hot non-natal osmotic environments in nature. However, at present, their habitat range is considered mainly from a geographical point of view, and the limits of environmental parameters such as the temperature and salinity of their habitats are not clear. As a next step, field studies focusing on the temperature and salinity variations of their natural habitats should be conducted to determine the effect of the physiological limitations that were suggested from this study on their habitat selection in nature.

We conducted the experiments under extreme conditions to determine the limits of their osmoregulatory capacity. Tests under milder brackish conditions would contribute to a more detailed understanding of their osmoregulatory capacity in relation to temperature. This study was conducted during cool seasons to facilitate the maintenance of low temperature. Conducting similar experiments in warm seasons will be an 
Fig. 4 Survival rate of Japanese black porgy Acanthopagrus schlegelli acclimated to non-natal (hypotonic environment, a) and natal (seawater, b) osmotic environments under various temperature conditions. Blue, red and gray indicate cool, hot and control groups, respectively
(a) non-natal (hypotonic environment)

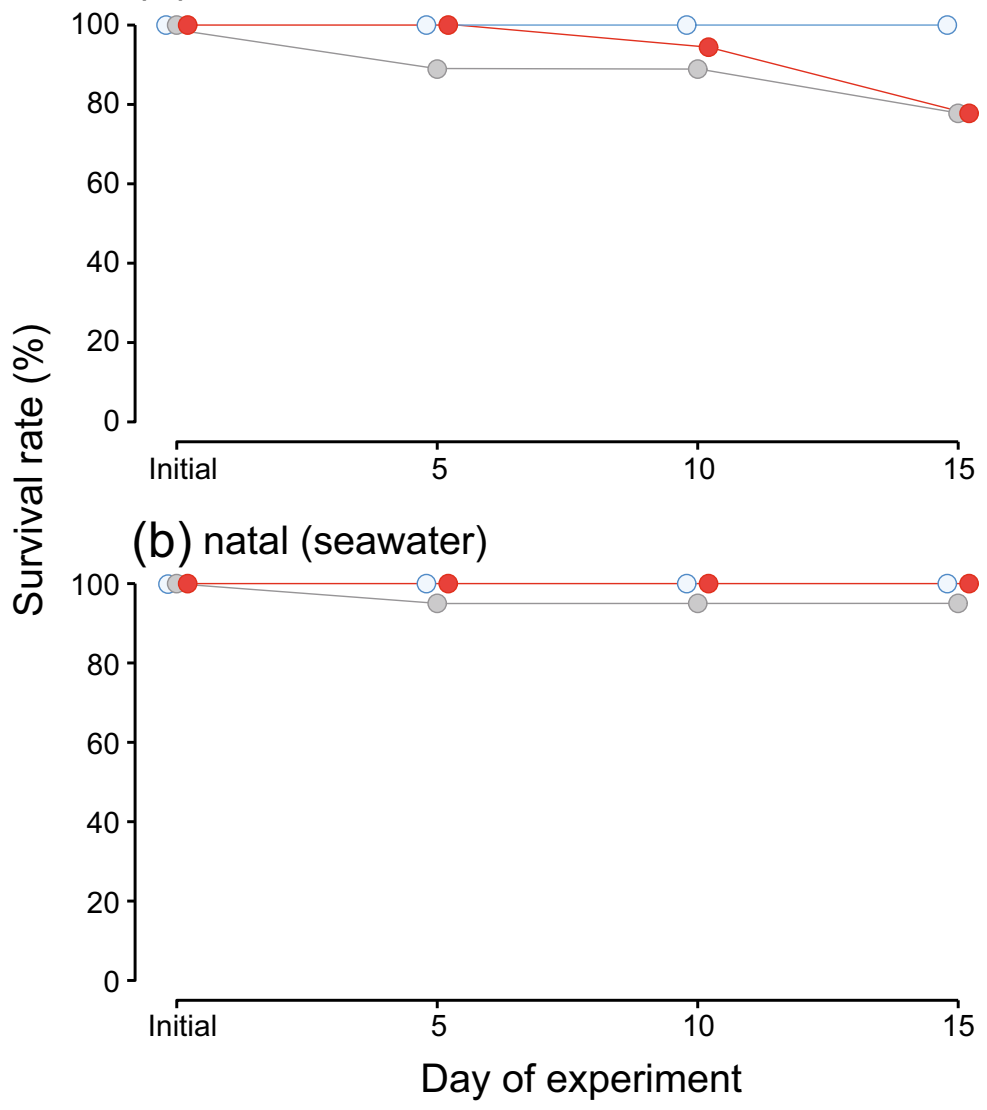

interesting approach to examine the seasonal effect on euryhaline wanderers' capacity to acclimate to salinity and temperature. These approaches will contribute to a more precise estimate of the effect of coastal warming on the migration ecology of these species.

\section{Conclusion}

This study strongly suggests that dace and black porgy are able to acclimate to higher temperatures in their natal osmotic environments than in non-natal osmotic environments. The fact that the two phylogenetically distant species have the same characteristics sheds light on the possibility that the osmoregulatory capacities of various euryhaline wanderers under high temperature conditions may be maintained in their natal osmotic environments, but are impaired in non-natal osmotic environments. This indicates that global warming may affect their seasonal habitat transitions. Further studies should be conducted on various euryhaline wanderer species to verify the generality of the characteristics suggested in this study and to precisely estimate the effect of coastal warming on the migratory ecology of these species.

Acknowledgements This study was funded by a Sasakawa Scientific Research Grant from the Japan Science Society to MN (26-741). We sincerely thank K. Takahashi (Keio University) for technical support in collection and rearing of fishes. We truly thank A. Hayashi (Seikai National Fisheries Research Institute) for advice on the statistical analysis. We are also grateful to M. J. Miller (Nihon University) and Y. Nakamura for helping to improve the manuscript. The experiments were conducted according to the principles and procedures approved by the Institutional Animal Care and Use Committee of the University of Tokyo.

\section{Compliance with ethical standards}

Conflict of interest The authors declare that they have no conflict of interest. 
Open Access This article is licensed under a Creative Commons Attribution 4.0 International License, which permits use, sharing, adaptation, distribution and reproduction in any medium or format, as long as you give appropriate credit to the original author(s) and the source, provide a link to the Creative Commons licence, and indicate if changes were made. The images or other third party material in this article are included in the article's Creative Commons licence, unless indicated otherwise in a credit line to the material. If material is not included in the article's Creative Commons licence and your intended use is not permitted by statutory regulation or exceeds the permitted use, you will need to obtain permission directly from the copyright holder. To view a copy of this licence, visit http://creativecommons.org/licenses/by/4.0/.

\section{References}

Able KW, Fahay MP (1998) The first year in the life of estuarine fishes in the middle Atlantic bight. Rutgers University Press, New Brunswick

Able KW, Fahay MP (2010) Ecology of estuarine fishes. The Johns Hopkins University Press, Baltimore

Ahokas RA, Sorg G (1977) The effect of salinity and temperature on intracellular osmoregulation and muscle free amino acids in Fundulus diaphanus. Comp Biochem Physiol A 56:101105. https://doi.org/10.1016/0300-9629(77)90448-0

Bennett WA, Beitinger TL (1997) Temperature tolerance of the sheepshead minnow, Cyprinodon variegatus. Copeia 1997: 77-87 https://www.jstor.org/stable/1447842

Bennett WA, Judd FW (1992) Comparison of methods for determining low temperature tolerance: experiments with pinfish, Lagodon rhomboides. Copeia 1992:1059-1065 https://www. jstor.org/stable/1446638

Day JW, Hall CAS, Kemp WM, Yanez-Arancibia A (1989) Estuarine ecology. Wiley, New York City

Finstad B, Staurnes M, Reite OB (1988) Effect of low temperature on sea-water tolerance in rainbow trout, Salmo gairdneri. Aquaculture 72:319-328. https://doi.org/10.1016/00448486(88)90220-7

Fujita S (2004) Ecological study on larvae and juveniles of the two sea basses and the three sparines occurring in the Shimanto estuary, Japan. Bull Mar Sci Fish Kochi Univ 23:1-57 (in Japanese with English abstract)

Imsland AK, Gunnarsson S, Foss A, Stefansson SO (2003) Gill $\mathrm{Na}^{+}, \mathrm{K}^{+}$-ATPase activity, plasma chloride and osmolality in juvenile turbot (Scophthalmus maximus) reared at different temperatures and salinities. Aquaculture 218:671-683. https://doi.org/10.1016/S0044-8486(02)00423-4

Ishitobi Y, Hiratsuka J, Kuwabara H, Yamamuro M (2000) Comparison of fish fauna in three areas of adjacent eutrophic estuarine lagoons with different salinities. J Mar Syst 26: 171-181. https://doi.org/10.1016/S0924-7963(00)00052-X

Ishizaki D, Otake T, Sato T, Yodo T, Yoshioka M, Kashiwagi M (2009) Use of otolith microchemistry to estimate the migratory history of Japanese dace Tribolodon hakonensis in the Kamo River, Mie prefecture. Nippon Suisan Gallaishi 75:419-424 (in Japanese with English abstract). https://doi.org/10.2331/suisan.75.419

Katano O, Nakamura T, Abe SI, Baba Y (2010) Population density, growth and migration via the sea to different streams of
Japanese dace Tribolodon hakonensis in lower reaches of small streams. Ichthyol Res 57:1-9

Lima FP, Wethey DS (2012) Three decades of high-resolution coastal sea surface temperatures reveal more than warming. Nat Commun 3:704. https://doi.org/10.1038/ncomms1713

Masroor W, Farcy E, Gros R, Lorin-Nebel C (2018) Effect of combined stress (salinity and temperature) in European sea bass Dicentrarchus labrax osmoregulatory processes. Comp Biochem Physiol A Mol Integr Physiol 215:45-54. https://doi.org/10.1016/j.cbpa.2017.10.019

Metz JR, Van Den Burg EH, Bonga SEW, Flik G (2003) Regulation of branchial $\mathrm{Na}^{+} / \mathrm{K}^{+}$-ATPase in common carp Cyprinus carpio L. acclimated to different temperatures. J Exp Biol 206:2273-2280. https://doi.org/10.1242/jeb.00421

Moriyama T, Fujisaku M, Mizutani M, Goto A (2008) Migration of Japanese dace (Ugui) in water zone network formed by streams, canal and river utilized for irrigation and drainage - a case study of Nishikinugawa district, Tochigi prefecture. Trans of JSIDRE 254:1-10 (in Japanese with English abstract)

Nakamura M, Masuda R, Tsukamoto K, Otake T (2016) Narrowed temperature adaptability in non-natal osmotic environments of two euryhaline wanderers, dace and black porgy: implications for seasonal habitat changes. Fish Sci 82:261-268. https://doi.org/10.1007/s12562-015-0956-6

R Core Team (2017) R: a language and environment for statistical computing. R Foundation for Statistical Computing. https://www.R-project.org/. Accessed 14 June 2017

Sakai H (1995) Life-histories and genetic divergence in three species of Triborodon (Cypriniodae). Memoirs of the Faculty of Fisheries Sciences, Hokkaido University 42:1$98 \mathrm{http} / /$ hdl.handle.net $/ 2115 / 21893$

Sardella BA, Kültz D, Cech JJ, Brauner CJ (2008) Salinitydependent changes in $\mathrm{Na}^{+} / \mathrm{K}^{+}$-ATPase content of mitochondria-rich cells contribute to differences in thermal tolerance of Mozambique tilapia. J Comp Physiol B 178: 249-256. https://doi.org/10.1007/s00360-007-0211-2

Tran TT, Tran HD, Kinoshita I (2019) Simultaneous and sympatric occurrence of early juveniles of Acanthopagrus latus and A. schlegelii (Sparidae) in the estuary of northern Vietnam. Limnology 2019:1-6. https://doi.org/10.1007/s10201-01900581-3

Tsutsumi E, Guo X (2016) Climatology and linear trends of seasonal water temperature and heat budget in a semienclosed sea connected to the Kuroshio region. JGR Oceans 121:4649-4669. https://doi.org/10.1002/2016JC011748

Umminger BL (1971) Patterns of osmoregulation in freshwater fishes at temperatures near freezing. Physiol Zool 44:20-27 https://www.jstor.org/stable/30155548

Vargas-Chacoff L, Regish AM, Weinstock A, McCormick SD (2018) Effects of elevated temperature on osmoregulation and stress responses in Atlantic salmon Salmo salar smolts in fresh water and seawater. J Fish Biol 93:550-559. https://doi.org/10.1111/jfb.13683

Yokoo T, Mito T, Iwasaki T, Sasaki T, Michine T, Aranishi F (2010) Spatial and temporal variation of fish assemblages surveyed by set-nets in Nakaumi Lagoon. Jpn J Limnol 71:11-18 (in Japanese with English abstract). https://doi.org/10.3739/rikusui.71.11

Publisher's note Springer Nature remains neutral with regard to jurisdictional claims in published maps and institutional affiliations. 